\title{
Influence of Cultural Factors in Dynamic Trust in Automation
}

\author{
Shih-Yi Chien, Michael Lewis \\ School of Information Sciences \\ University of Pittsburgh \\ Pittsburgh, PA 15260 USA \\ shc56@pitt.edu,ml@sis.pitt.edu
}

\author{
Katia Sycara \\ Robotics Institute \\ Carnegie Mellon University \\ Pittsburgh, PA 15213 USA \\ katia@cs.cmu.edu
}

\author{
Jyi-Shane Liu \\ Dept. of Computer Science \\ National ChengChi University \\ Taipei, Taiwan \\ jsliu@cs.nccu.edu.tw
}

\author{
Asiye Kumru \\ Dept. of Psycholoy \\ Özyeğin University \\ Istanbul, Turkey \\ asiye.kumru@ozyegin.edu.tr
}

\begin{abstract}
The use of autonomous systems has been rapidly increasing in recent decades. To improve human-automation interaction, trust has been closely studied. Research shows trust is critical in the development of appropriate reliance on automation. To examine how trust mediates the human-automation relationships across cultures, the present study investigated the influences of cultural factors on trust in automation. Theoretically guided empirical studies were conducted in the U.S., Taiwan and Turkey to examine how cultural dynamics affect various aspects of trust in automation. The results found significant cultural differences in human trust attitude in automation.
\end{abstract}

Keywords-Trust in Automation; Human Factors; Culture; Humancomputer Interaction; Reliability; Workload; User Study

\section{INTRODUCTION}

Automation is increasingly being used in all aspects of our interactions with computers and other machinery. Increasingly powerful and complex automation, however, may not work perfectly under various boundary conditions. Because automated systems inevitably involve uncertainty, trust, as an intervening variable, is related to the calibration between the user's expectations and the automation capabilities. Studies have shown trust significantly influences human decision making in uncertain or risky conditions [1], [2]. As favorable behaviors and outcomes are provided by the automation, for example, the operator tends to increase reliance on the automated systems. Therefore, human trust in automation is dynamic, and external factors (e.g., task contexts) exert a meaningful influence over the trust decisions.

\section{A. Trust in Automation}

Trust has been studied in various disciplines, in which research shows trust is dynamic and affected by the experience of the system. When interacting with a system, prolonged interaction with the automation leads an operator to make generalizations about the automation and develop the operator's belief about the future behaviors of the system. In other words, early in the relationship, the trust in the system is primarily based on the predictability of the system's behavior. Previous studies [3], [4] have shown shifts in trust in response to changes in properties and performance of the automation. When the automation performed reliably, operator trust increased over time and vice versa. As trust decreased, the automation might be ignored or switched off and manual control became more frequent.

\section{B. Cultural Mechanisms}

Culture is an important modulator, which contributes at different stages in the development of trust decisions [5]-[7]. Prior work [8] investigated psychological as well as behavioral outcomes of individuals from different cultures using Hofstede's cultural dimensions [9]. A more recent theory, cultural syndromes [10] categorizes cultural characteristics into three types, Dignity, Honor and Face cultures. Cultural syndromes examine how cultural norms affect an individual's behaviors in ways complementary to Hofstede cultural dimensions.

\section{1) Hofstede's Cultural Dimensions}

Three of the cultural constructs developed by Hofstede were adopted for our study.

Power distance (PD) refers to "the extent to which the less powerful accept and expect that power is distributed unequally [9]," in which the high PD society expects the less powerful members to obey and accept authority.

Individualism (IDV) is "the degree of interdependence a society maintains among its members [9]." For example, a member from a high IDV society tends to pursue individual achievements, instead of group goals.

Uncertainty avoidance (UA) is defined as "the extent to which the members of a culture feel threatened by uncertain or unknown situations [9]," in which low UA represents better tolerance for uncertainty and ambiguity.

\section{2) Cultural Syndromes}

Three groups of cultures have been characterized through the cultural syndromes.

Dignity cultures emphasize individuality with members assessing their own self-worth. Dignity cultures are widespread in Western Europe and North America and lead to societies where interpersonal transactions are governed by law. Because of the expectation that another will behave according to law, an individual from a dignity culture will have higher initial trust and tend to trust others until proven otherwise.

Face cultures found in East Asia, are based on a stable social hierarchy and strong social norms. People in Face cultures cherish in-group harmony and value others views of her/him. Trust is high for those within the group and low for those outside. 
Honor cultures found in Middle East and Latin America. Due to the unstable social hierarchy, the member of an honor culture in general has low interpersonal and institutional trust and requires longer experience to develop trust.

These cultural mechanisms, Hofstede cultural dimensions and cultural syndromes, were adopted in our study to formulate research hypotheses and examine how cultural factors may affect trust in automation. For example, Honor culture operators should take longer interaction times than operators from Dignity and Face cultures to develop an equal degree of trust in automation.

The goal of the present cross-cultural study was to identify and describe the ways cultural differences affect cognition and behavior. Experimental tasks were therefore designed to elicit as wide a range of previously reported effects as possible. A substantial portion of trust in automation studies [2] have been limited to simple target detection tasks with decision support systems of varying reliability. Lee and Moray [4] used a monitoring task with simulated discrete failures while assessing the operators' confidence. Effects were measured on a rating of trust and resulting behaviors. Dzindolet [11] used an automatic target recognition task manipulating automation imperfection in order to measure effects on operator confidence. While the insights that were provided by these early works were seminal, many documented effects on automation reliance require a more complex environment. To better measure the factors affecting trust in automation, an air traffic control simulation, was adapted for our study to simulate a variety of forms of autonomy in a dynamic multi-agent multitask environment.

\section{METHODS}

Data for this study was collected in the U.S., Taiwan and Turkey. These countries were selected based on two sets of criteria. The first criterion was the differences they exhibit on the most well studied Hofstede cultural dimensions (e.g. the U.S. is high on IDV, Turkey is medium, and Taiwan is low; the U.S. is low on PD whereas Turkey is high, with Taiwan in medium; the U.S. is low on UA whereas Taiwan is medium and Turkey is high). Second, each of these countries belongs to a different cultural syndrome; U.S. (Dignity), Taiwan (Face) and Turkey (Honor).

\section{A. Apparatus}

The experiments were conducted by modifying the existing air traffic control simulator, Research Environment for Supervisory Control of Heterogeneous Unmanned Vehicles (RESCHU) [12]. Five simulated unmanned air vehicles (UAVs) were used in the experiments. RESCHU provided a map display for navigation, payload window for target search, message box for assigned missions, UAV status window for vehicles' current statuses, and mission timeline for the ongoing and upcoming tasks (Figure 1).

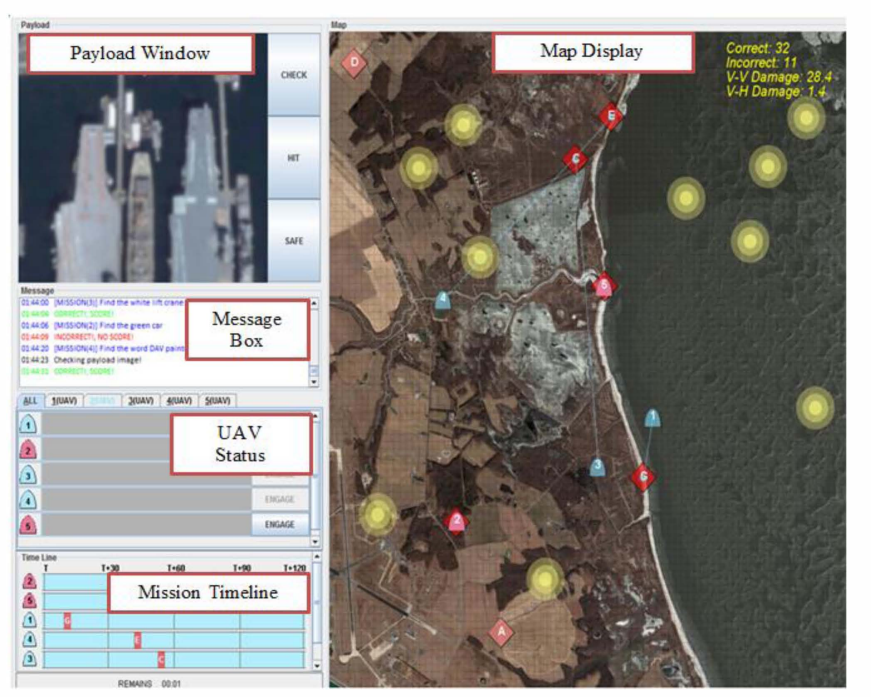

Figure 1. RESCHU user interface. The map window shows the numbered UAVs (blue half ovals) with paths to targets (red diamonds); threat areas are marked by the yellow circles. While an UAV reaches a target, the engage button will be switched on and the UAV icon will begin flashing.

\section{B. Experimental Tasks}

The experimental tasks involved identifying an assigned enemy target and attacking it (payload task), and rerouting the UAVs' paths if necessary (navigation task).

\section{1) Experimental Tasks I: Payload Tasks}

Vehicles were routed to areas of suspected target(s). When an UAV reached a target area, a series of actions needed to be performed to acquire sufficient situation awareness to accomplish the payload tasks. Upon arrival at the target area the operator was presented with a panorama in the payload window and asked to search for a specific object (red squares in Figure $2 \mathrm{a} \& 2 \mathrm{~b}$ ) within the image. The operator first observed a low-resolution picture (Figure 2a), along with three options: Check, Hit and Safe. By clicking the "Check" button, after a three-second delay, the system provided the picture with higher resolution so the operator could identify the target with greater certainty (Figure 2b).

If an operator could not find the assigned hostile target, the operator could choose the Safe mode to proceed with other tasks, otherwise Hit would be chosen to attack the target. Following payload submission, the UAV was assigned to another available target and the process repeated. Meanwhile, the message box notified the operator whether the submission was correct or not (green and red texts on the message box).

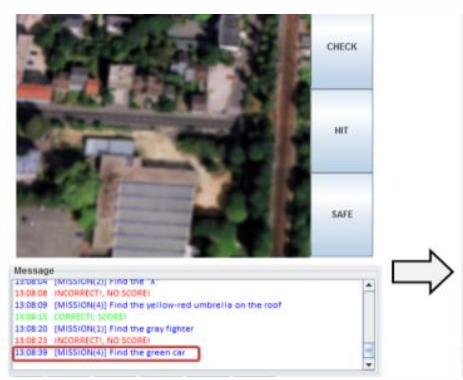

(2a) Before checking

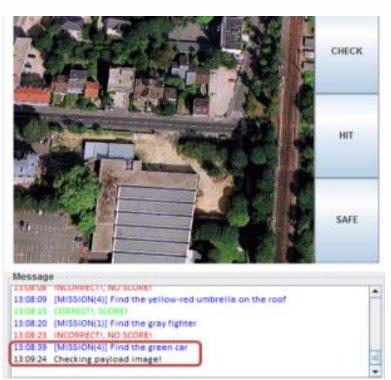

(2b) After checking
Figure 2. Target detection tasks. 


\section{2) Experimental Tasks II: Navigation Tasks}

While performing multi-UAV tasks, one of the operator's most commonly encountered problems is the path conflict. In our experiment, the UAVs' paths were generated by an autonomous path planner following a shortest distance criterion, in which each UAV had limited sensor range and different respective targets. The navigation tasks required an operator to detect and avoid threat areas (yellow dots on Fig. $3 a)$ and reroute the UAV's conflicted path (Fig. 3b). The operator must add waypoints to avoid the collisions or go around the threat areas.

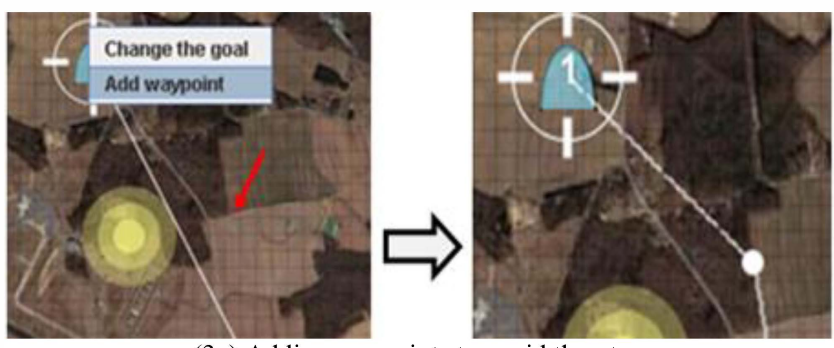

(3a) Adding waypoints to avoid threat areas

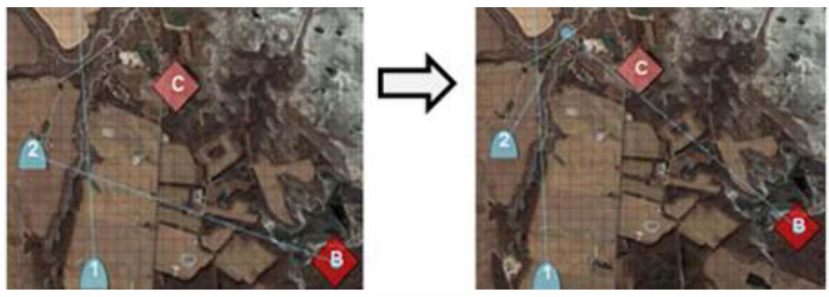

(3b) Adding waypoints to avoid path conflicts

Figure 3. Adding vehicle's waypoints to avoid collisions.

\section{Automated Assistance}

Two types of automated assistance were provided to assist the operators. The target finder marked the possible targets on the images in the payload tasks. In the navigation tasks the conflict detector highlighted the path conflicts and hazards and/or rerouted the UAVs to avoid the collisions.

\section{1) Automated Assistance I: Target Finder}

The likelihood alarm system (LAS) was used by the target finder in order to examine the effects of operators' uncertainty avoidance. LAS generated three types of cues to provide information about the likelihood of the critical events to focus operators' attention on suspicious conditions [13], [14].

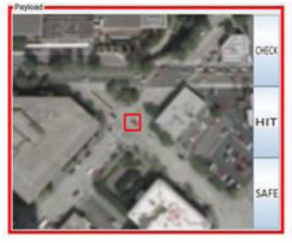

(4a) Alarm

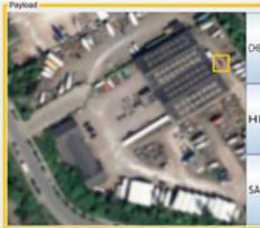

(4b) Warning

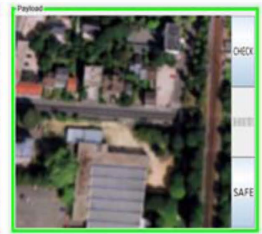

(4c) non-alert
Figure 4. Three types of cues in target finder.

Based on the automated diagnosis, the payload window was highlighted in different colors and a bounding box was added on top of the suspected target (Fig. 4a \& 4b). A red border represented the alarm condition (Fig. 4a), which had high likelihood to truly indicate the assigned target; whereas the warning condition was represented by a yellow color (Fig. 4b), which was associated with a higher level of uncertainty, informing the operator that there might be an assigned target. A green border indicated anon-alert condition (Fig. 4c), which had relatively low possibility that the assigned target was included in the image; however, with the non-alert cue, if the operator detected a target, she needed to manually add a square on the suspected target to activate the Hit mode (Fig. 5).

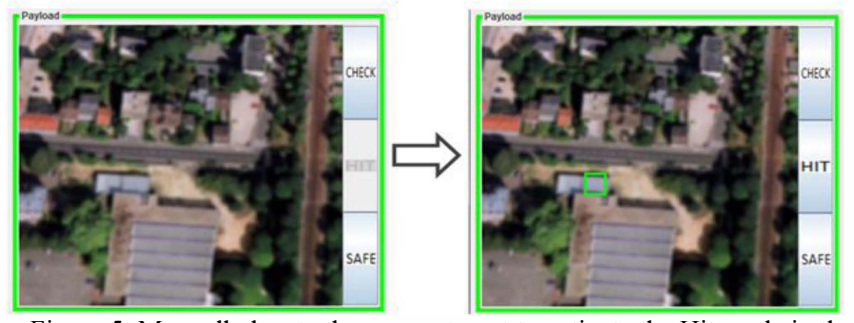

Figure 5. Manually locate the enemy target to activate the Hit mode in the non-alert condition.

\section{2) Automated Assistance II: Conflict Detector-Path Re-plan}

To prevent multi-UAV collisions, a collision avoidance algorithm was adopted to resolve the conflicts among the UAVs. Given the path information, the conflict detector suggested new paths and diverted the involved UAVs from the collision course before appropriate separation was violated. By clicking the Auto button (Fig. 6), the involved UAVs were directed from the normal (straight) paths to curve paths, by which the UAVs could reach their desired destination with minimum path/time under the additional constraints (collision avoidance).
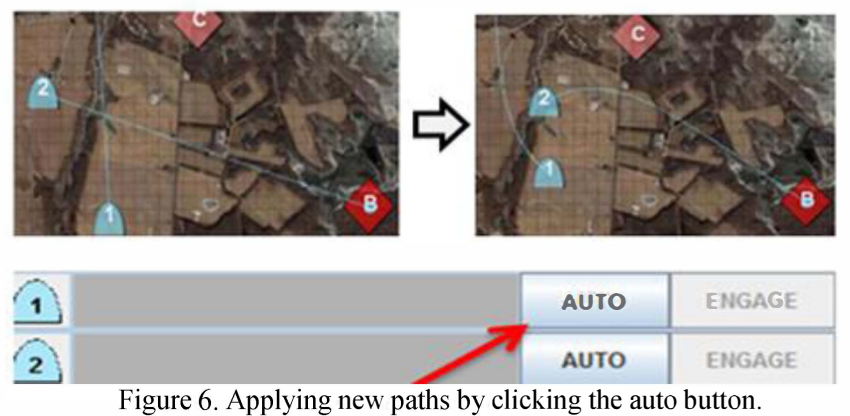

\section{3) Automated Assistance III: Conflict Detector-Highlights}

The transparency of automation, the operator's understanding of its behavior, can greatly affect the degree of perceived difficulty in using it [2]. The path re-planning mechanism can be opaque because it acts to prevent collisions that have not occurred and may not yet be apparent. Based on the same UAV path information used by the path re-planner, the highlighting mechanism drew a red square on the map to highlight the path conflicts between the UAVs (Fig. 7a) and red dots if the UAV travelled through the threat areas (Fig. 7b).

\section{Experimental Design}

To investigate participants' trust in automation, reliability of the target finder and conflict detector were manipulated as well as task load to control the major factors previously found to affect trust. 


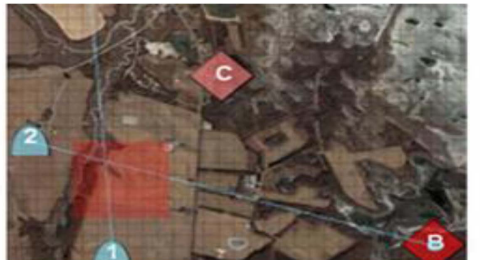

(7a) Path conflicts

Figure 7. Highlighting the conflicts and threat areas.

\section{1) Source Reliability in Target Finder and Conflict Detector}

To simulate the effect of imperfect automation, the source reliability of the target finder and conflict detector were varied.

In target finder, the reliability for the alarm conditions (red boarder in Figure 4a) and non-alert condition (green boarder in Figure 4c) were $80 \%$ across all experimental conditions, whereas in the warning condition (yellow color in Figure 4b) the reliability was either high $(80 \%)$ or low $(20 \%)$. Additionally, the reliability of the conflict detector was $90 \%$ across all experimental conditions (i.e., 1 out of 10 suggested path was unnecessary). Inappropriate reliance on faulty suggestions from the conflict detector would increase the UAVs' travel time to the targets.

\section{2) Task Load}

Instead of changing the number of controlled UAVs, the task load was manipulated through changes to the UAVs' speed. The speed doubled between conditions, in which the vehicles moved at 2.5 pixels/second in the low task load condition and 5.0 pixels/second in the high-load condition.

\section{E. Experimental Conditions}

The experiment followed a mixed repeated measures design, with source reliability in target finder (high-80\% vs. low$20 \%$ ), automation transparency in conflict detector (path replan, highlight, path re-plan $x$ highlight), and countries (U.S., Taiwan and Turkey) as the between-subject variables, and task load (high vs. low) as the within factor.

Table 1. Experimental Conditions.

\begin{tabular}{|c|c|c|c|c|}
\hline $\begin{array}{c}\text { Conflict Detector } \\
\text { B/t Varriables } \\
\text { Target Finder }\end{array}$ & $\begin{array}{c}\text { Control } \\
\text { Condition }\end{array}$ & Path Re-plan & Highlight & $\begin{array}{c}\text { Path Re-plan } \\
\text { x Highlight }\end{array}$ \\
\hline $\begin{array}{c}\text { High } \\
\text { Reliability }\end{array}$ & HRC & HRP & HRH & HRPH \\
\hline Low & \multicolumn{4}{|c|}{$\begin{array}{r}\text { With-in variables } \\
\text { (High/Low task load) }\end{array}$} \\
Reliability & LRC & LRP & LRH & LRPH \\
\hline
\end{tabular}

\section{F. Participants and Procedures}

The study was conducted in the U.S., Turkey and Taiwan. As the experimental conditions shown in Table 1, each cell included 15 participants; 120 participants $\left(15^{*} 8=120\right)$ were recruited in each country and data from a total of 360 participants $(120 * 3=360)$ were collected from the three countries. The student participants were recruited from the University of Pittsburgh in the U.S., National Chengchi University in Taiwan, and Ozyegin University in Turkey. None had prior experience with air traffic control although most were frequent computer users.
Prior to beginning, participants were asked to complete the trust instrument we developed in earlier research [15], [16], Big Five inventory [17] and CVSCALE [18] measuring initial trust in automation, personality traits, and cultural characteristics respectively. Participants then took an interactive training tutorial (approximately 20 minutes) to practice control operations and, based on the randomly assigned condition, learn all aspects of the simulation with the assisted applications (i.e., target finder or/and conflict detector). Participants were informed that the automation was fairly but not perfectly reliable. The goal was to identify and attack as many hostile targets as possible and avoid path conflicts while engaging as many targets as possible. After completing the training tasks, participants began the first 10-minute experimental session in which they performed the target classification and path planning tasks by controlling 5 UAVs. At the conclusion of the session participants completed the trust instrument to evaluate their trust in the specific uses of the automated assistance(s) and NASA-TLX workload survey [19] to measure the task load. After a brief break, the other task load condition was run accompanied by repeated trust questionnaire and workload survey. To avoid the language issues, Chinese and Turkish versions of instruments were also used in our study.

The reported trust score was obtained from our trust questionnaire, in which general trust was characterized by system performance, process, purpose, and task contexts; whereas the automation specific measure only included three factors (performance, process and purpose). Details about these scales and their development can be found in [15], [16].

\section{RESULTS}

A mixed-model ANOVA was used to determine the differences in trust, where country (U.S., Taiwan and Turkey), source reliability in target finder (high: $80 \%$ vs. low: 20\%), and automation transparency in conflict detector (path re-plan, highlight, and path re-plan $\mathrm{x}$ highlight) were the betweensubject variables, and task load (high vs. low) was the withinsubject factor.

\section{A. Initial/General Trust}

The analyses revealed significant cultural effects on initial trust in automation $\left(\mathrm{F}_{2,357}=15.012, \mathrm{p}<.001\right)$. The U.S. participants rated the highest score in general trust and the Turkish group was the lowest with the Taiwanese rates falling in between, Fig. 8. Pairwise T-tests showed significant differences between U.S. and Taiwanese $(p=.048)$, U.S. and Turkish $(\mathrm{p}<.001)$, and Taiwanese and Turkish $(\mathrm{p}=.007)$ groups.

\section{B. Specific Trust}

To measure the specific use of automation under a variety of situations, the conditions of experiencing task load, source reliability in target finder, and automation transparency in conflict detector were manipulated. 


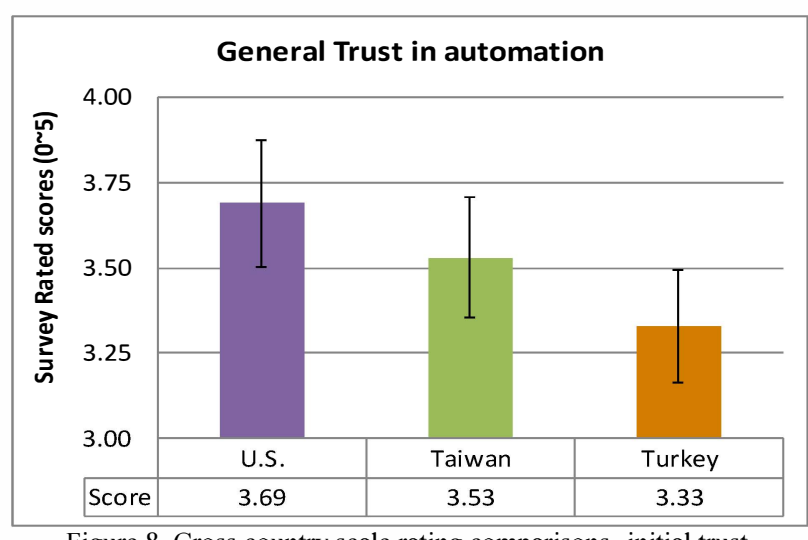

Figure 8. Cross country scale rating comparisons- initial trust.

\section{1) Task Load}

While increasing the UAVs' speed, no difference was observed on trust in the conflict detector with regard to the navigation tasks. However, in terms of the trust in target finder, the analysis showed participants had significantly higher trust $(\mathrm{p}=.002)$ in the high workload (HW) than low workload (LW) condition (Fig. 9). A pairwise T-test found a significant difference between task load conditions in Taiwanese $(p=.008)$ as well as Turkish $(\mathrm{p}=.002)$ participants, in which significantly higher trust score was rated under HW situation; however no statistical differences were found in the U.S. group with regard to workload.

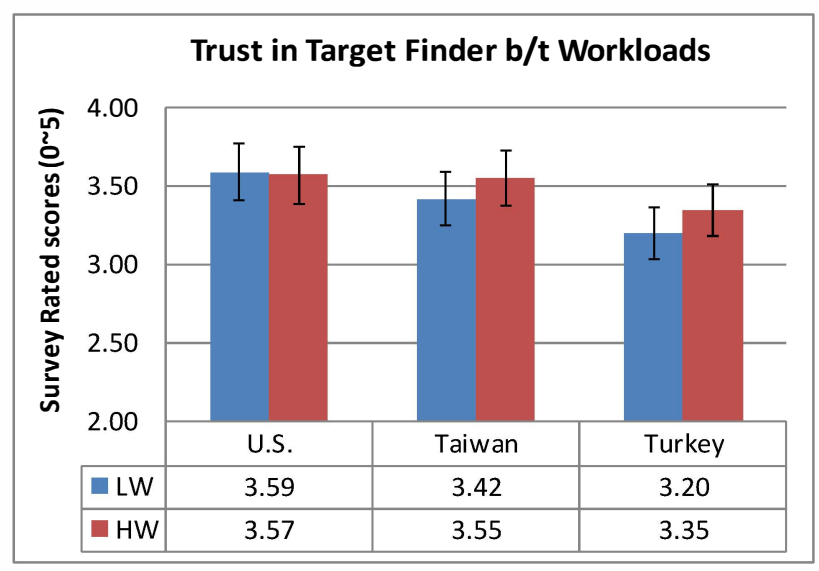

Figure 9. Specific trust in target finder in different workload.

\section{2) Source Reliability- Target Finder}

Two levels of source reliability were tested for the target finder (High-80\% vs. Low-20\%). The analysis found significant effects for country $\left(\mathrm{F}_{4,706}=5.997, \mathrm{p}<.001\right)$ and reliability conditions $\left(\mathrm{F}_{2,353}=9.854, \mathrm{p}<.001\right)$, Fig. 10 . Increasing the source reliability led to significantly higher trust scores. Further analysis (T-test) revealed a significant difference between the U.S. and Turkish group in both high reliability $(\mathrm{HR})$ condition $(\mathrm{p}<.001)$ and low reliability (LR) condition $(p<.001)$. The analysis also revealed a significant difference between Taiwanese and Turkish population $(p=.001)$ in the HR condition and a marginal difference $(p=.089)$ in the LR condition. No difference was observed between U.S. and Taiwanese participants.

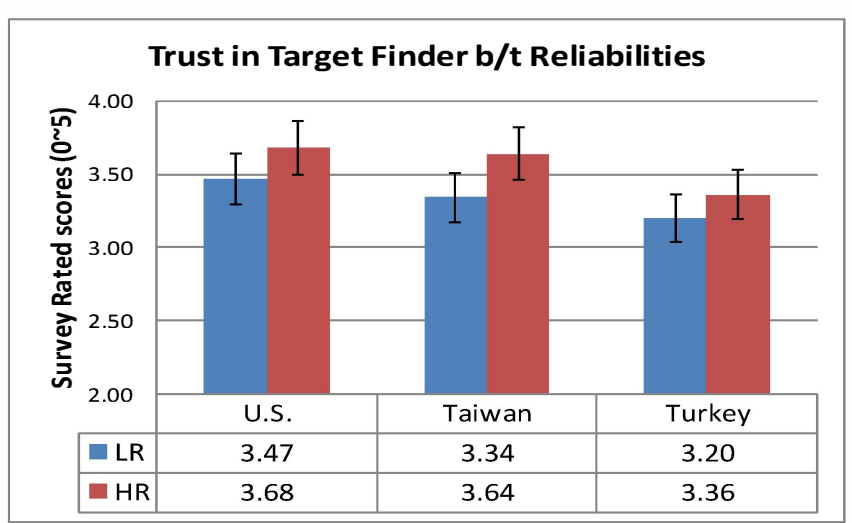

Figure 10. Specific trust in target finder in different reliability

\section{3) Automation Transparency-Conflict Detector}

To examine the influences of automation transparency, three conflict detector conditions were tested, path re-plan (PR), highlight (HL), and path re-plan $\mathrm{x}$ highlight (PRHL), to assist operators to detect the possible path conflicts between UAVs. The analysis revealed significant differences for automation transparency $\left(\mathrm{F}_{4,520}=5.709, \mathrm{p}<.001\right)$ and, again, country $\left(\mathrm{F}_{4,520}=5.399, \mathrm{p}<.001\right)$, Fig. 11 .

Operators had the highest trust while interacting with HL condition and the lowest rates in the PR condition with PRHL falling in between. A pairwise T-test found differences between HL and PR $(p<.001)$, PRHL and PR $(p<.001)$, and HL and PRHL $(p=.010)$. Additionally, significant cultural differences were also found between U.S. and Turkey, and Taiwan and Turkey in both PR ( $p=.016$ and $p<.001$ respectively) and PRHL conditions ( $p=.001$ and $p<.001$ respectively), in which the trust scores in U.S. and Taiwanese population were higher than the Turkish group. No effects were found in the HL condition among the three cultures. Pairwise T-test showed consistent differences between HL and PR, and PRHL and PR across the three cultures. It is worth mentioning the difference between HL and PRHL was only observed in Turkish population, in which the trust score in HL was significantly higher than PRHL.

\section{DISCUSSION AND FUTURE WORK}

The present study examined the influence of cultural factors on trust in automation. The theoretically guided experimental studies were conducted in the U.S., Taiwan and Turkey to empirically determine how cultural factors affect various aspects of trust and reliance on automation. Due to the page limit, this report focused only on differences between cultural factors and trust ratings. The other measures (such as performance in target detection, etc.) remain to be described elsewhere.

\section{A. General Trust}

As hypothesized according to cultural syndromes, the member of an honor culture has lower level of initial trust whereas an individual from Dignity culture has a higher level of general trust. In other words, honor culture operators should require longer interaction times than operators from Dignity and Face cultures to develop an equal degree of trust in 
automation. These cultural effects were confirmed, in which the U.S. group (dignity) had the highest score in general trust and Turkish participants (honor) were the lowest, with Taiwan population (face) falling in between.

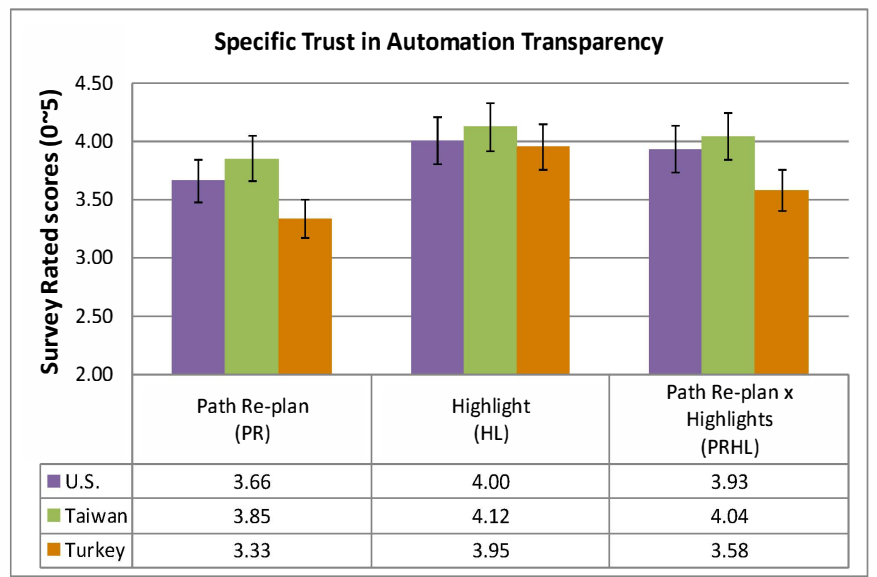

Figure 11. Trust in automation transparency across cultures.

\section{B. Task Load}

Increasing task load required operators to allocate more attention to the navigation tasks and therefore less resource was reserved for the payload tasks. An interesting finding was observed, in which increasing the UAVs speed failed to exert influences on trust in the navigation tasks, but indirectly affected trust in the payload task (target finder).

The results suggested operators had higher trust in the target finder under heavy task load conditions. This could have resulted because little attention was allocated to the automated recommendations from the target finder causing the operators to fail to scrutinize the provided suggestions. In addition, the effect of task load significantly influenced the Taiwanese and Turkish populations but was not observed in the U.S. group. Although the Taiwanese and Turkish cultures differ substantially, a recent study [20] showed that for some forms of behavior Face (Taiwan) and Honor (Turkey) cultures are closely related, which can explain the non-significant statistical differences.

\section{Source Reliability}

With increased reliability, as expected, participants had higher trust in the automated assistance. In addition, as predicted by cultural syndromes, honor culture operators should be the slowest when compared to Face and Dignity operators to repair trust after automation failure. These effects were also confirmed in our study, in which the U.S. and Taiwanese group rated significantly higher trust in both LR and HR conditions than the Turkish population.

\section{Automation Transparency}

Automation transparency was found to have significant effects on trust in specific automation. Higher trust was found in the HL group than the PR and PRHL conditions. However, only the Turkish group showed a significant difference between HL and PRHL conditions. Instead of automatically generating new paths for UAVs, the HL condition only provided collision detection and required the operators to manually prevent the possible conflict. Because Honor cultures have low initial trust and require longer interaction time to establish trust, this type of human automation interaction was especially beneficial to the individuals from Honor cultures.

Future work will include further examinations on trust dissolution after the occurrence of faults and trust restoration of trust in automation.

\section{ACKNOWLEDEMENTS}

This research has been sponsored by AFOSR FA9550-13-1-0129.

\section{REFERENCES}

P. A. Hancock, D. R. Billings, K. E. Schaefer, J. Y. C. Chen, E. J. de Visser, and R. Parasuraman, "A Meta-Analysis of Factors Affecting Trust in Human-Robot Interaction," Hum. Factors J. Hum. Factors Ergon. Soc., vol. 53, no. 5, pp. 517-527, Sep. 2011

[2] J. Lee and K. See, "Trust in automation: designing for appropriate reliance.," Hum. Factors, vol. 46, no. 1, pp. 50-80, Jan. 2004.

[3] B. Muir, "Trust in automation: Part I. Theoretical issues in the study of trust and human intervention in automated systems," Ergonomics, vol. 37, no. 11, pp. 1905-1922, 1994.

[4] J. Lee and N. Moray, "Trust, control strategies and allocation of function in human-machine systems," Ergonomics, 1992.

[5] M. Brewer and R. Kramer, "The psychology of intergroup attitudes and behavior," Annu. Rev. Psychol., 1985.

[6] I. Bohnet, B. Herrmann, and R. Zeckhauser, "The Requirements for Trust in Gulf and Western Countries," Q. J. Econ., vol., 2009.

[7] C. A. Fulmer and M. J. Gelfand, "Dynamic Trust Processes: Trust Dissolution and Restoration," pp. 1-32, 2010

[8] Merritt, "Culture in the Cockpit: Do Hofstede's Dimensions Replicate?," J. Cross. Cult. Psychol., 2000.

[9] Hofstede, "Cultures And Organizations - Software of the Mind," Development, pp. 1-29, 1991.

[10] H. Triandis, "The psychological measurement of cultural syndromes.," Am. Psychol., vol. 51, no. 4, pp. 407-415, 1996.

[11] M. T. Dzindolet, L. G. Pierce, H. P. Beck, and L. a. Dawe, "The Perceived Utility of Human and Automated Aids in a Visual Detection Task," Hum. Factors J. Hum. Factors Ergon. Soc., 2002.

[12] Y. Boussemart and M. Cummings, "Behavioral recognition and prediction of an operator supervising multiple heterogeneous unmanned vehicles," Humans Oper. unmanned, 2008.

[13] C. Wickens and A. Colcombe, "Dual-task performance consequences of imperfect alerting associated with a cockpit display of traffic information.," Hum. Factors, 2007.

[14] R. Wiczorek and D. Manzey, "Supporting Attention Allocation in Multitask Environments: Effects of Likelihood Alarm Systems on Trust, Behavior, and Performance," Hum. Factors J. Hum. Factors Ergon. Soc., vol. 56, pp. 1209-1221, 2014.

[15] S. Chien, M. Lewis, S. Hergeth, Z. Semnani-Azad, and K. Sycara, "Cross-Country Validation of a Cultural Scale in Measuring Trust in Automation," Hum. Factors Ergon. Soc. Annu. Meet., 2015.

[16] S. Chien, Z. Semnani-azad, M. Lewis, and K. Sycara, "Towards the Development of an Inter-cultural Scale," 2014.

[17] O. John and S. Srivastava, "The Big Five trait taxonomy: History, measurement, and theoretical perspectives," Handb. Personal. Theor, no. 510, 1999 .

[18] B. Yoo, N. Donthu, and T. Lenartowicz, "Measuring Hofstede's five dimensions of cultural values at the individual level: Development and validation of CVSCALE," J. Int. Consum. Mark., vol. 23, no. August 2011, pp. 193-210, 2011.

[19] S. G. Hart and L. E. Staveland, "Development of NASA-TLX (Task Load Index): Results of empirical and theoretical research," in Human Mental Workload, vol. 1, P. A. Hancock and N. Meshkati, Eds. North-Holland, 1988, pp. 139-183.

[20] S. Aslani, J. Ramirez-Marin, Z. Semnani-Azad, J. M. Brett, and C. Tinsley, Dignity Face and Honor cultures implications for negotiation and conflict management. 2013, p. 561. 